\title{
Topographic rectification in a stratified ocean
}

\author{
by K. H. Brink ${ }^{1}$
}

\begin{abstract}
Mean flow generation by oscillating currents is considered in an idealized stratified ocean with a corrugated shelf-slope topography. Numerical model results for near-bottom mean along-isobath flow show a general parameter dependence similar to that found with no stratification (Brink, 2010). Stratification, however, makes the interior rectified flow depth-dependent, and usually bottom-intensified. Scalings for both the mean flow and its vertical dependence are developed and evaluated. Two crude comparisons against observed mean oceanic flows suggest that the present results may parameterize the mean flow magnitude correctly.
\end{abstract}

\section{Introduction}

Oscillating barotropic flow across straight isobaths generates, through tidal rectification, a mean along-isobath velocity, provided that either there is some (albeit vanishingly small) dissipation in the system (e.g., Huthnance, 1973; Stern and Shen, 1976; Loder, 1980) or that the oscillations have finite amplitude (e.g., Visser, 1994). When the isobaths are not straight, the resulting mean flow becomes weaker as the topographic irregularity scale decreases (Brink, 2010: B10 henceforth). B10's numerical model experiments are summarized by a scaling that accounts for a good deal of the mean flow energy, but that has enough scatter as not to be valid on a point-by-point basis. For representative oceanographic conditions (oscillatory speeds of about $0.1 \mathrm{~m} / \mathrm{s}$, slopes of 0.01-0.001, topographic scales of 10-50 km, water depth $100 \mathrm{~m}$ ), these scalings for barotropic topographically induced mean currents suggest that the resulting flow in a realistic setting is perhaps $\mathrm{O}(0.01-0.1 \mathrm{~m} / \mathrm{s})$ or less.

Scalings for the mean flow generated over variable topography in a forced, dissipative system bear some resemblance (especially in terms of dependence on water depth) to expressions for the mean flow that occurs in the seemingly different situation of an eddy field evolving adiabatically over irregular topography (e.g., Merryfield et al., 2001). B10 conjectures that the similarities between the two disparate models' results are not a coincidence, but that it is essentially an expression of the same duality that occurs between

1. Department of Physical Oceanography, Woods Hole Oceanographic Institution, Woods Hole, Massachusetts, 02543, U.S.A.email: kbrink@whoi.edu 
small-amplitude dissipative, and large-amplitude inviscid tidal rectification with straight isobaths.

One might ask how the inclusion of continuous density stratification affects the B10 forced, frictional topographic rectification model. Some informative adiabatic ("Neptune") models have included stratification in a quasi-geostrophic context (e.g., Merryfield, 1998), but these results are not valid for a general parameter range, and they correspond to a case with no energy source or sink. Maas and Zimmerman (1989a,b) address the dissipative tidal rectification problem with stratification, straight isobaths and spatially uniform fluctuating flow. Their results are, however, limited to the case where depth changes are small, and where the damping is weak. Nonetheless, their results highlight important aspects of the resulting mean flow, especially bottom trapping and the secondary circulation (in the crossisobath/vertical plane). Bottom trapping is a natural result of density stratification inhibiting vertical motions, and its vertical extent is readily estimated from scaling a vorticity equation. For example, Merryfield et al. (2001) use such a vorticity scaling, and conjecture a hyperbolic cosine vertical structure if constant density stratification were included in their barotropic adiabatic model with finite topography. With regard to the secondary circulation, especially interesting results have been obtained using realistic, two-dimensional, finite amplitude primitive equation numerical model runs, e.g., Chen and Beardsley (1995). Thus, previous results show that topographic rectification still occurs in the presence of density stratification, but that its effects would be concentrated near the bottom boundary.

The next step in studying topographic rectification thus appears to be the inclusion of density stratification for a problem that includes forcing, dissipation and variable, finiteamplitude topography. This step is a straightforward extension of B10, but is essential for improving the scaling's applicability to the real ocean. Among the questions to be addressed here are whether Merryfield et al.'s conjecture about the vertical structure is correct, whether a mean-flow scaling based on extending the barotropic approach is valid, and whether the magnitude of the resulting mean flow with stratification is similar to that for the barotropic case. The present approach follows that of B10: dynamically based scalings for mean flow and its structure are used to encapsulate the results from multiple numerical model runs. The calibrated scalings are then compared to a small sample of observed oceanic mean flow measurements.

\section{Methodology}

\section{a. Model configuration}

The Regional Oceanic Modeling System (ROMS: Shchepetkin and McWilliams, 2005) primitive equation numerical model is used throughout this study. It solves the equations

$$
\begin{aligned}
u_{t}+u u_{x}+v u_{y}+w u_{z}-f v & =-\rho_{0}^{-1} p_{x}+\left(A u_{z}\right)_{z}, \\
v_{t}+u v_{x}+v v_{y}+w v_{z}+f u & =-\rho_{0}^{-1} p_{y}+\left(A v_{z}\right)_{z}, \\
u_{x}+v_{y}+w_{z} & =0
\end{aligned}
$$




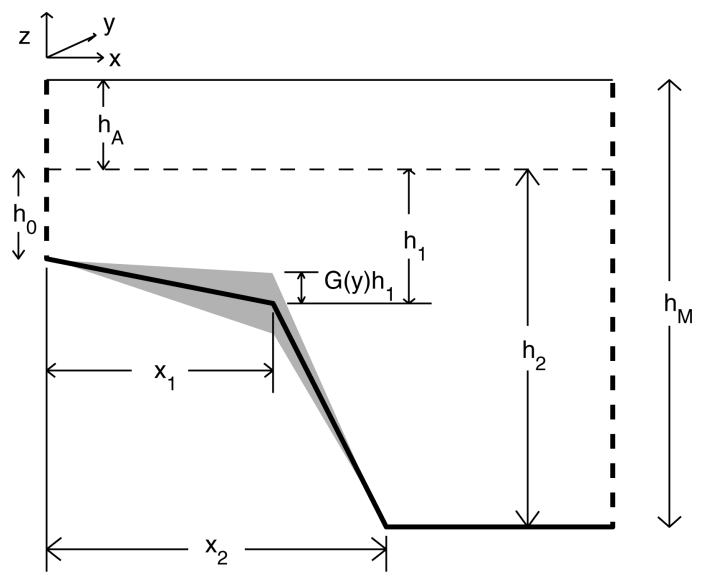

Figure 1. Definition sketch of the model geometry.

$$
\begin{aligned}
T_{t}+u T_{x}+v T_{y}+w T_{z} & =\left(K T_{z}\right)_{z}, \\
\rho & =\rho_{0}\left[1-\alpha\left(T-T_{0}\right)\right] .
\end{aligned}
$$

The velocity components $(u, v, w)$ are in the $(x, y, z)$ directions, where $z$ is the vertical coordinate (positive upwards). The temperature is $T$ and the density is $\rho$, while the equation of state $(1 \mathrm{e})$ is linearized about the base state $\left(T_{0}, \rho_{0}\right)$ with thermal expansion coefficient $\alpha$. The constant Coriolis parameter is $f$, and $t$ is the time variable. Subscripts $(x, y, z, t)$ represent partial differentiation. The bottom boundary condition is given by

$$
A v_{z}=c_{D}|v| v
$$

where $c_{D}$ is a drag coefficient. In contrast, B10 uses a linearized bottom stress: the present choice is driven by compatibility with the Brink and Lentz (2010a,b) bottom boundary layer results. The comparative effects of linearized vs. quadratic bottom stress for a barotropic tidal rectification problem are treated by Wright and Loder (1988). The eddy coefficients $A$ and $K$ are found using the Mellor-Yamada level 2.5 closure scheme (e.g., Wijesekera et al., 2003). No lateral mixing is applied in any of the model runs.

The model is configured as a cyclic (in the $y$, alongshore, direction) channel, with open onshore and offshore boundaries. The bottom topography (Figs. 1, 2) is the same as in B10: a two-slope configuration adjoining a deep, flat-bottom ocean:

$$
\begin{array}{lr}
h(x, y)=h_{A}+h_{0}+C x+h_{1} G(y) x / x_{1} & \text { for } 0<x<x_{1} \\
h(x, y)=h_{A}+h_{1}+B\left(x-x_{1}\right)+h_{1} G(y)\left(x_{2}-x\right) /\left(x_{2}-x_{1}\right) & \\
h(x, y)=h_{2}=h_{A}+h_{1}+B\left(x_{2}-x_{1}\right) & \text { for } x_{1}<x<x_{2} \\
& \text { for } x_{2}<x
\end{array}
$$



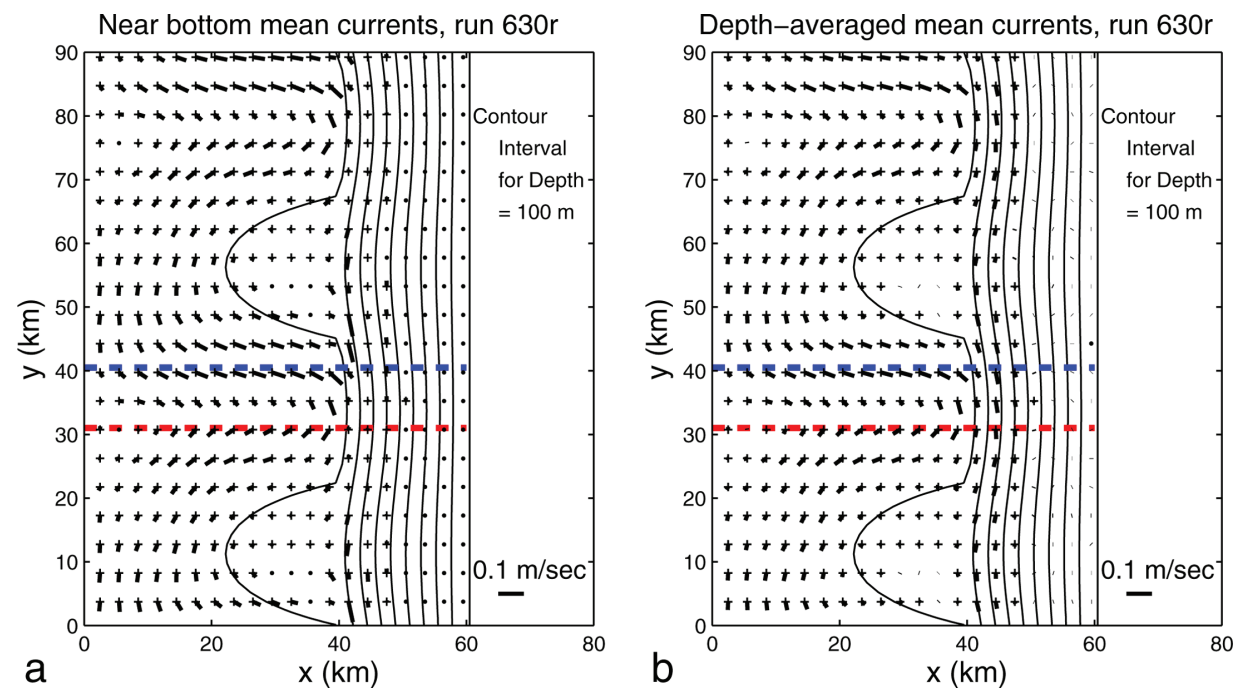

Figure 2. (a) Plan view of mean currents for run 630 at the lowest elevation outside the bottom boundary layer. The light contours are water depth (contour interval $=100 \mathrm{~m}$ ). Velocities are shown as heavy lines, oriented so that there is a "plus" sign at the base of the vector, i.e., the flow is away from the plus sign. The blue and red dashed lines are the locations of the cross-shelf sections shown in Figure 3. For this run, the average bottom boundary layer thickness is $21 \mathrm{~m}$ for the locations that are plotted. Results from every third point in both $x$ and $y$ are plotted. (b) The same as Figure 2(a), except depth-averaged velocity is shown.

where $h_{1}=h_{0}+C x_{1}$ and $h_{A}$ is a constant. In most cases, $x_{1}=40 \mathrm{~km}, x_{2}=60 \mathrm{~km}$, $h_{A}=0, h_{0}=25 \mathrm{~m}, h_{1}=100 \mathrm{~m}$, and $h_{2}=1000 \mathrm{~m}$. The overall depth is characterized in Table 1 by the maximum depth, $h_{M}$. Topographic irregularity enters through the function $G(y)$, viz.,

$$
G(y)=\sum_{n} a_{n} \sin \left(k_{n} y\right),
$$

where usually only a single term is used. The amplitude of the topographic irregularities is indicated in Table 1 by the standard deviation of depth at the shelf break (where the depth has its maximum alongshore variability), $h_{C}$. The model grid spacing is $1 \mathrm{~km}$ in $x$ and $1.5 \mathrm{~km}$ or finer (depending on the $k_{n}$ ) in $y$. In the vertical, 30 levels are used, with the grid points distributed so as to resolve the bottom boundary layer relatively well. The ROMS stretching parameters $\theta_{S}=4.0, \theta_{B}=1.0$ and Tcline $=40 \mathrm{~m}$ are used and these lead, for example, to vertical resolutions of $1.7-5.5 \mathrm{~m}$ in $100 \mathrm{~m}$ of water, and 2.3 to $12.4 \mathrm{~m}$ in $200 \mathrm{~m}$ of water, with the finer resolutions in the boundary layers.

In B10, forcing was provided through a fluctuating alongshore surface wind stress. This approach is impractical for the stratified problem because near-surface mixing would often completely eliminate stratification over much of the shelf. Adding a stabilizing surface heat 
Table 1. The following parameters are included here. The Coriolis parameter is $f, c_{D}$ is the bottom frictional drag coefficient, $h_{M}$ is the maximum water depth, $\lambda$ is the alongshore wavelength of the bottom topography, $h_{C}$ is standard deviation of water depth at the shelf break, $\tau$ is the forcing period, $u_{I}$ is the amplitude of the imposed inflow at the shallower boundary, $T_{z}$ is the initial vertical temperature gradient, $\Xi$ is the normalized topostrophy (a measure of the tendency for flow to follow isobaths: Holloway, 2008), "Mult" is the maximum degree of frequency multiplication in the response, and $v_{M M}$ is the strongest time-mean velocity in the model domain.

\section{Numerical Model Run Summary}

\begin{tabular}{|c|c|c|c|c|c|c|c|c|c|c|c|}
\hline $\begin{array}{l}\text { Run } \\
\text { Number }\end{array}$ & $\begin{array}{c}f \\
\left(1 / \mathrm{s} \times 10^{4}\right)\end{array}$ & $\begin{array}{c}c_{D} \\
\left(\times 10^{3}\right)\end{array}$ & $\begin{array}{l}h_{M} \\
(\mathrm{~m})\end{array}$ & $\begin{array}{c}\lambda \\
(\mathrm{km})\end{array}$ & $\begin{array}{l}h_{C} \\
(\mathrm{~m})\end{array}$ & $\begin{array}{c}\tau \\
\text { (days) }\end{array}$ & $\begin{array}{c}u_{I} \\
(\mathrm{~m} / \mathrm{s})\end{array}$ & $\begin{array}{c}T_{z} \\
(\% / \mathrm{m})\end{array}$ & $\Xi$ & Mult & $\begin{array}{l}v_{M M}^{\dagger} \\
(\mathrm{m} / \mathrm{s})\end{array}$ \\
\hline $610^{*}$ & 1.0 & 2.9 & 1000 & 45 & 59 & 4 & 0.05 & 0.005 & 0.43 & 3 & -0.12 \\
\hline $611^{*}$ & 1.0 & 2.9 & 1000 & 45 & 59 & 4 & 0.05 & 0.01 & 0.44 & 3 & -0.14 \\
\hline $612^{*}$ & 1.0 & 2.9 & 1000 & 45 & 59 & 4 & 0.05 & 0.02 & 0.46 & 3 & -0.15 \\
\hline $613^{*}$ & 1.0 & 2.9 & 1000 & 45 & 59 & 4 & 0.05 & 0.001 & 0.33 & 3 & -0.12 \\
\hline $620^{*}$ & 1.0 & 2.9 & 1000 & 45 & 59 & 8 & 0.05 & 0.01 & 0.18 & 5 & -0.12 \\
\hline $621^{*}$ & 1.0 & 2.9 & 1000 & 45 & 59 & 2 & 0.05 & 0.01 & 0.28 & 2 & -0.14 \\
\hline $622^{*}$ & 1.0 & 2.9 & 1000 & 45 & 59 & 0.75 & 0.05 & 0.01 & 0.12 & 1 & -0.02 \\
\hline 623 & 1.0 & 2.9 & 1000 & 90 & 59 & 8 & 0.05 & 0.01 & 0.19 & $\mathrm{C}$ & -0.16 \\
\hline $630^{*}$ & 1.0 & 6.0 & 1000 & 45 & 59 & 4 & 0.05 & 0.01 & 0.36 & 3 & -0.09 \\
\hline $631^{*}$ & 1.0 & 1.0 & 1000 & 45 & 59 & 4 & 0.05 & 0.01 & 0.34 & 4 & -0.18 \\
\hline $632^{*}$ & 1.0 & 6.0 & 1000 & 45 & 59 & 8 & 0.05 & 0.01 & 0.30 & 4 & -0.12 \\
\hline 633 & 1.0 & 6.0 & 1000 & 45 & 59 & 16 & 0.05 & 0.01 & 0.32 & $\mathrm{C}$ & -0.13 \\
\hline $635^{*}$ & 1.0 & 2.9 & 1000 & 45 & 59 & 4 & 0.10 & 0.01 & 0.31 & 3 & -0.18 \\
\hline 636 & 1.0 & 2.9 & 1000 & 45 & 59 & 4 & 0.20 & 0.01 & 0.40 & $\mathrm{C}$ & -0.31 \\
\hline $637^{*}$ & 1.0 & 2.9 & 1000 & 45 & 59 & 4 & 0.10 & 0.02 & 0.30 & 3 & -0.17 \\
\hline $662^{*}$ & 1.0 & 2.9 & 2000 & 45 & 79 & 4 & 0.05 & 0.005 & 0.40 & 1 & -0.30 \\
\hline $663^{*}$ & 1.0 & 2.9 & 1500 & 45 & 59 & 4 & 0.05 & 0.005 & 0.45 & 1 & -0.30 \\
\hline $664^{*}$ & 1.0 & 2.9 & 2000 & 45 & 79 & 4 & 0.05 & 0.01 & 0.27 & 1 & -0.33 \\
\hline $670^{*}$ & 1.0 & 2.9 & 1000 & - & 0 & 4 & 0.05 & 0.01 & 0.17 & 1 & -0.03 \\
\hline 671 & 1.0 & 2.9 & 1100 & 45 & 148 & 4 & 0.05 & 0.01 & 0.43 & $\mathrm{C}$ & -0.34 \\
\hline 672 & 1.0 & 2.9 & 1000 & 45 & 40 & 4 & 0.05 & 0.01 & 0.40 & 2 & -0.14 \\
\hline 673 & 1.0 & 2.9 & 1000 & 45 & 20 & 4 & 0.05 & 0.01 & 0.22 & 2 & -0.11 \\
\hline $675^{*}$ & 1.0 & 2.9 & 1000 & 10 & 59 & 4 & 0.05 & 0.01 & 0.43 & 4 & -0.11 \\
\hline $676^{*}$ & 1.0 & 2.9 & 1000 & 90 & 59 & 4 & 0.05 & 0.01 & 0.20 & 3 & -0.10 \\
\hline 677 & 1.0 & 2.9 & 1000 & $90,45,30$ & 85 & 4 & 0.05 & 0.01 & 0.35 & 4 & -0.16 \\
\hline 678 & 1.0 & 2.9 & 1000 & $90,45,30$ & 85 & 4 & 0.05 & 0.005 & 0.33 & 5 & -0.17 \\
\hline 679 & 1.0 & 2.9 & 1000 & 15 & 59 & 4 & 0.05 & 0.01 & 0.27 & 2 & -0.13 \\
\hline $680^{*}$ & 0.5 & 2.9 & 1000 & 45 & 59 & 8 & 0.05 & 0.01 & 0.10 & 3 & -0.08 \\
\hline $681^{*}$ & 0.5 & 6.0 & 1000 & 45 & 59 & 8 & 0.05 & 0.01 & -0.02 & 4 & -0.06 \\
\hline $682^{*}$ & 0.5 & 2.9 & 1000 & 45 & 59 & 8 & 0.05 & 0.02 & 0.01 & 2 & -0.09 \\
\hline $683^{*}$ & 1.0 & 2.9 & 1000 & 90 & 59 & 4 & 0.05 & 0.02 & 0.23 & 3 & -0.13 \\
\hline $684^{*}$ & 1.0 & 2.9 & 1000 & 30 & 59 & 4 & 0.05 & 0.01 & 0.27 & 2 & -0.11 \\
\hline 690 & 1.0 & 6.0 & 1000 & 45 & 59 & 8 & 0.10 & 0.02 & 0.04 & $\mathrm{C}$ & -0.19 \\
\hline $691^{* \dagger}$ & 1.0 & 2.9 & 1000 & 45 & 59 & 4 & 0.05 & 0.01 & 0.56 & 3 & -0.16 \\
\hline
\end{tabular}

"Mult": Frequency multiplier. "2" means that the response has frequency doubling, "3" tripling, "C" chaotic behavior, and " 1 " means that the response is at the forcing frequency at all locations.

* Runs used to evaluate scalings.

$\dagger$ Topography is compressed in the cross-shelf direction.

\$The strongest mean downwave velocity (as a function of both horizontal and vertical position). 
flux might help, but would raise other complications arising from variable stratification. For the present problem, forcing is provided by means of the depth-averaged flow through the offshore and onshore boundaries. That is,

$$
\begin{aligned}
\langle u\rangle & =u_{0}\left\{\frac{h(L, y)+\varsigma(L, y, t)}{h(0, y)+\varsigma(0, y, t)}\right\} \sin (\omega t) & \\
& \equiv u_{I} \sin (\omega t) & \text { at } x=0 \\
\langle u\rangle & =u_{0} \sin (\omega t) & \text { at } x=L
\end{aligned}
$$

where $\langle\ldots\rangle$ represents a vertical average, $\zeta$ is the free surface elevation (small compared to $h), \omega$ is the forcing angular frequency, and the forcing is ramped up smoothly over an initial 4-day interval. In all cases, $L=90 \mathrm{~km}$. This approach does not generate any near-surface mixing, and its cross-shelf flow pattern can be thought of as equivalent to the subsurface flow that compensates for surface Ekman transport during wind-driven upwelling or downwelling (i.e., the interior flow that balances surface Ekman transport when a solid wall is encountered). The model is run for 96 days or longer, so that the initial transients are entirely damped out. Runs that are obviously "chaotic" (meaning that the flow does not repeat from one forcing cycle to the next) are excluded from consideration in the statistical calculations because they did not establish consistent mean flows. Most of the runs used in the following calculations, however, have some degree of frequency multiplication in their response (Table 1) as might be expected in a mean-flow generation problem (e.g., Loder, 1980). For nonchaotic runs, model mean (averaged over a forcing period) flow must be everywhere steady to within the larger of $0.01 \mathrm{~m} / \mathrm{s}$ or $10 \%$ of the maximum value, and this requirement sometimes requires longer model runs.

\section{b. The bottom boundary layer}

In the barotropic problem (B10), the bottom boundary layer was not resolved, and all results could be discussed in terms of the depth-averaged flow. In the present problem, the flow is distinctly depth-dependent both in the interior, and especially in the turbulent bottom boundary layer. The model's bottom boundary layer is often about 20-m thick or more, and its structure is largely as expected from stratified, oscillating flow over a sloping bottom (Brink and Lentz, 2010b). However, for present purposes, only the velocity above the turbulent bottom boundary layer is of interest. Since tens of thousands of points are used for the evaluation of the mean flow scalings (Section 4), it is important to have an objective means to identify and eliminate points within the bottom boundary layer.

The following considerations are applied to eliminate bottom boundary points as well as to limit the number of locations used for evaluation.

a) All locations over a flat bottom in the offshore part of the domain are eliminated: this area does not generate a mean flow through topographic interaction. 
b) In locations with weak time-mean depth-averaged flow, mean velocity profiles as a function of $z$ are often dominated by vertical variability that has several extrema distributed over the water column. Because it is hard to resolve this pattern into interior and boundary layer signatures, locations with weak time- mean depth-averaged flow are deleted from further analysis. Specifically, for a given run, all locations are eliminated where the vector mean depth-averaged speed (i.e., the speed computed using the time mean of $\langle u\rangle$ and the time mean of $\langle v\rangle$ ) is less than $10 \%$ of the domain maximum. Because the deleted points have weak mean flow energy, their elimination is not expected to affect the evaluation of scalings (Section 4): these estimates would be dominated by higher-energy locations in any case.

c) For a bottom-intensified interior flow, as is generally found here, one expects that the mean speed is a maximum immediately above the frictional boundary layer. Within a given profile, a boundary layer depth is thus estimated as the deepest maximum of vector-mean speed. While this approach often gives reasonable results, vertical meanspeed profiles sometimes have multiple extrema ("wiggliness") near the bottom, so that knowing which speed maximum to pick is problematic. Experimentation shows that smoothing a profile with 5 passes of a "1-2-1" filter eliminates the smaller scale features and generally makes the height of the deepest smooth speed maximum a reasonable estimate of the boundary layer thickness.

d) In some cases, the above approach still yields unreasonably large, e.g., > $100 \mathrm{~m}$, bottom boundary layer thicknesses (experiment shows that these apparent thick boundary layers are not an artifact of the grid resolution). A theoretical maximum boundary layer thickness is then imposed, based on Brink and Lentz (2010b): the steady bottom boundary layer thickness for maximal down-slope Ekman transport:

$$
h_{B L}=\frac{v_{M} \Gamma}{N_{0} s}
$$

where

$$
\begin{aligned}
\Gamma & =\frac{1}{2}\left[1+\sqrt{1+4 R^{D} s^{2}}\right], \\
s & =|\nabla h| N_{0} / f,
\end{aligned}
$$

the buoyancy frequency $N_{0}$ is the initial, constant, interior buoyancy frequency, and $R^{D}=0.7$. The velocity $v_{M}$ at a given location is the maximum instantaneous speed obtained at any depth over the last forcing period: a decidedly conservative choice because the formula is meant to apply to a time-averaged velocity. The estimate $h_{B L}$ is then the maximum allowable boundary layer thickness at a given location.

Results of the boundary layer detection analysis are saved for consistent reference. For a given model run, the average bottom boundary layer thickness over the sloping bottom is typically of order $20 \mathrm{~m}$. Results of this analysis are shown in Figures $3 \mathrm{a}$ and $3 \mathrm{~b}$ as red contours marking the top of the bottom boundary layer. 

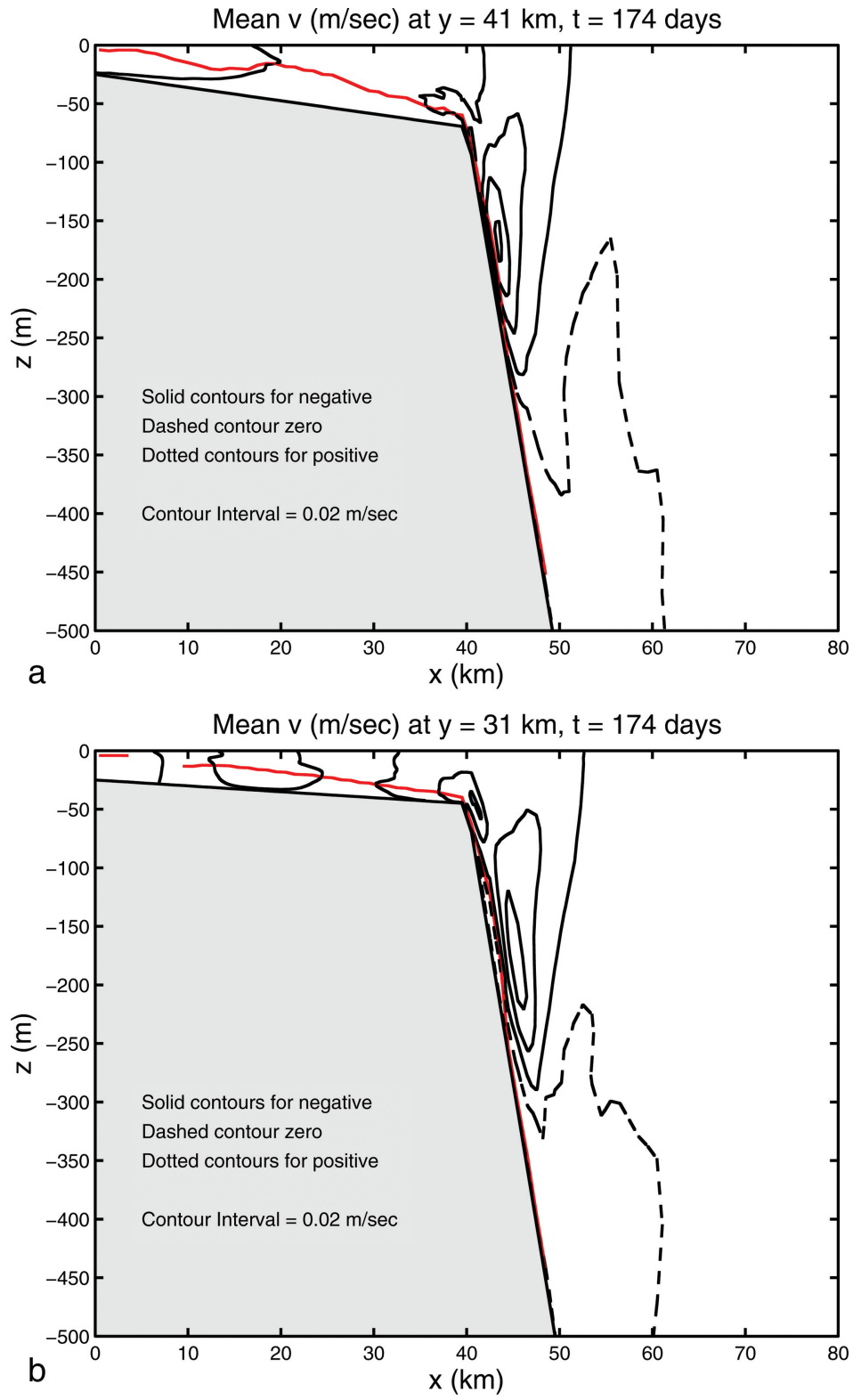

Figure 3. (a) Cross section of mean along-isobath velocity (positive for upwave flow) at $y=41 \mathrm{~km}$ for run 630. The location of the section is shown as a blue dashed line in Figure 2. The zero contour is dashed, positive contours would be dotted if they were present, and negative contours are solid lines. The contour interval is $0.02 \mathrm{~m} / \mathrm{s}$. The red contour represents the depth of the deepest grid point that is not in the bottom boundary layer. (b) Cross section of mean along-isobath velocity (positive for upwave flow) at $y=31 \mathrm{~km}$ for run 630 . The location of the section is shown as a red dashed line in Figure 2. The zero contour is dashed, positive contours would be dotted if they were present, and negative contours are solid lines. The contour interval is $0.02 \mathrm{~m} / \mathrm{s}$. The red contour represents the depth of the deepest grid point that is not in the bottom boundary layer. 


\section{Results}

A total of 34 numerical model runs are reported (Table 1), each running for at least 96 model days. In some cases, where it takes longer for a quasi-steady state to be reached, the model is run for up to 250 days in order to try to achieve repeatable flow patterns. In some cases (labeled "chaotic"), the response never settles in to a pattern that is repeatable from one forcing cycle to the next. These chaotic runs are excluded from any further statistical investigation.

As in the barotropic case (B10), the mean flow immediately above the bottom boundary layer generally follows isobaths (e.g., run 630: Fig. 2a). (This pattern might be contrasted with stratified flow over a pronounced, narrow submarine canyon where a closed circulation occurs in the embayment, e.g., She and Klinck, 2000). The most striking, but perhaps least surprising, difference between the stratified and barotropic rectification results is that with stratification, the time-mean flow decreases strongly with height above the bottom, especially over the continental slope (Fig. 3). The strongest flow is generally found at depths of about 130-180 $\mathrm{m}$ for this run, i.e., over the upper slope, and well below the surface. In the shallower shelf waters, any bottom trapping is less obvious and the mean flow appears fairly barotropic (i.e., having nearly vertical isotachs above the bottom boundary layer) relative to conditions farther offshore. Over the slope, velocity contours (e.g., $-0.02 \mathrm{~m} / \mathrm{s}$ in Fig. 3) are aligned neither vertically nor horizontally, but they tilt somewhat offshore with height above the bottom. This structure is similar to that of alongshore velocity in coastal-trapped waves with stratification and a sloping bottom, e.g., Huthnance (1978).

The continuity of the relatively strong mean flow just above the bottom boundary layer and near the shelf edge (Fig. 2a) deserves comment. In most locations (such as at $y=41 \mathrm{~km}$ : blue dashed line in Fig. 2a), the strongest mean flow occurs near the top of the bottom boundary layer (Fig. 3a), so a distinct along-isobath mean is evident in plan view (Fig. 2a) at that location. In locations where a substantial mean along-isobath flow is not found at the top of the bottom boundary layer (e.g., $y=31 \mathrm{~km}$ : Fig. 3b), the core current is still in the same depth range, is slightly weaker ( $-0.06 \mathrm{vs} .-0.08 \mathrm{~m} / \mathrm{s}$ for this example), and has meandered slightly offshore, so that the negative mean velocity core is now about $130 \mathrm{~m}$ above the bottom. Hence, the shelfbreak mean current appears to be fairly continuous, but this continuity is not very evident if attention is confined only to velocity immediately above the bottom boundary layer (as in Fig. 2a). Indeed, if one plots depth-averaged flow (Fig. $2 \mathrm{~b}$ ), the continuity of the shelfbreak current becomes much more obvious.

As in the barotropic case (B10), there are some clear statistical tendencies in the model outputs. For example, in nearly all cases, the standard deviation of along-isobath fluctuating velocity is larger than for cross-isobath velocity. Averaged over the 24 nonchaotic runs (denoted by asterisks in Table 1), the values just above the bottom boundary layer are 0.07 and $0.05 \mathrm{~m} / \mathrm{s}$, respectively. The mean along-isobath currents just above the bottom boundary layer are pattern-correlated with the standard deviation of cross-isobath flow, $S D\left(u_{\perp}\right)$ ( -0.32 for 33,941 grid points from the 24 runs). Further, there is also some tendency for this mean flow to follow isobaths: its correlation with $\nabla h / h$ is -0.13 , while the correlation 


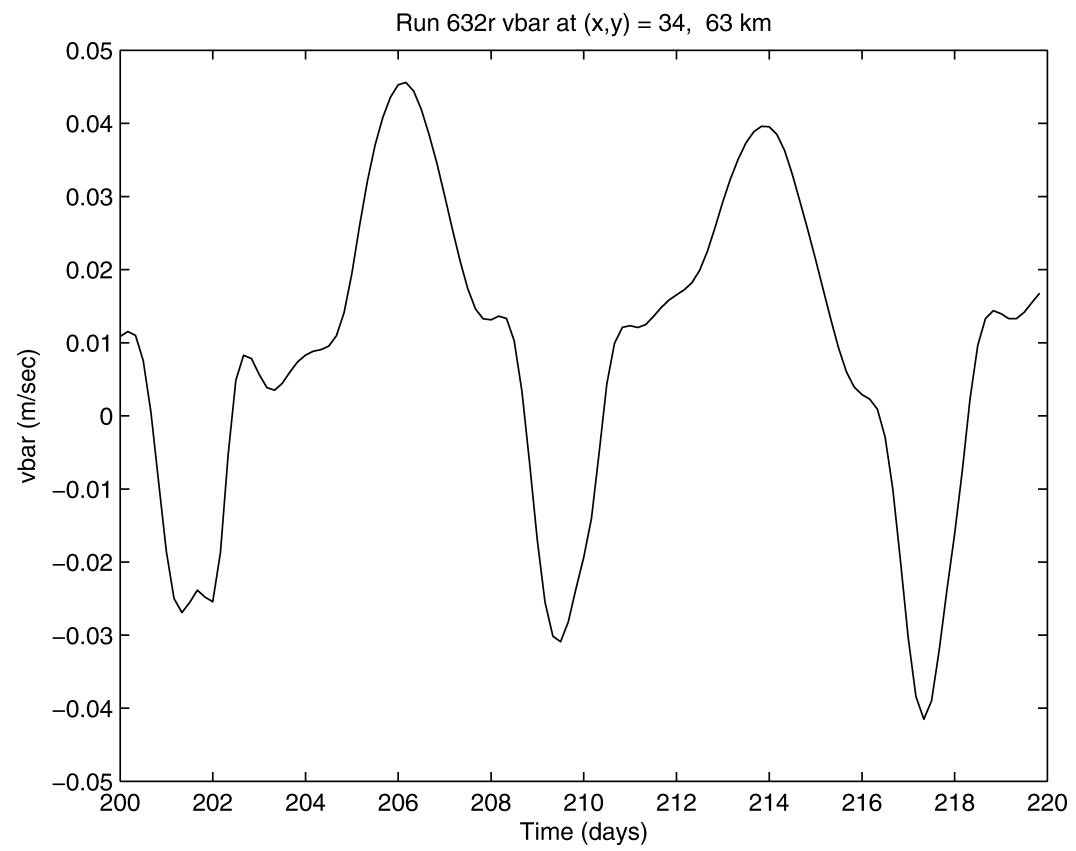

Figure 4. Depth-averaged alongshore ( $y$-direction) velocity for run 632 at $x=34 \mathrm{~km}, y=63 \mathrm{~km}$ (over the shelf). The forcing period is 8 days for this run.

with $S D\left(u_{\perp}\right) \nabla h / h$ is -0.33 over the same set of runs. The negative sense of the correlation indicates downwave mean flow (meaning flow in the direction that long topographic Rossby waves propagate). These correlations are to be expected based on the barotropic results (B10), and they are consistent with the scaling of Section 4.

The model response usually shows a good deal of temporal complexity. All model runs are inspected for locations of "chaotic" response, or of frequency multiplication (i.e., where there are multiple current maxima or minima during a single forcing cycle). Of the 34 runs, only 2 show no frequency multiplication or chaos, while 10 runs are chaotic (Figure 4 is an example from a run that is chaotic by the criterion of repeatability to within 1\%.) All of the other runs show frequency multiplication by a factor of 2-5. In the barotropic case (B10), it was possible to construct a regime diagram inspired by Samelson and Allen (1987) that differentiated where, in parameter space, a chaotic response is expected. In the present, stratified case, no comparably useful sorting is obtained. In particular, the amplitude of the forcing $u_{0}$ (nor any scaled version such as a Rossby number or Froude number) is not a useful predictor of the occurrence of chaos. The only useful rule is that no chaotic response is found for higher forcing frequencies, i.e. for $\omega \geq 0.04 \omega_{0}$ where $\omega_{0}$ is the deep-water internal Kelvin wave frequency, $\omega_{0}=2 \pi N h_{M} / \lambda$, where $\lambda$ is the dominant alongshore topographic wavelength. Other appropriate wave frequencies were also considered, but the 
Kelvin wave, or equivalently, the long, nonrotating, internal gravity wave speed, is the most effective at segregating runs. Presumably, the complexity of the present chaotic regime separation reflects the model's increased physical complexity, relative to the barotropic case.

\section{Scaling for mean along-isobath flow}

\section{a. Bottom-trapping}

Bottom trapping and vorticity conservation lead to the conjecture (as in Merryfield et al., 2001) that mean currents over a sloping bottom in a stratified ocean might have a vertical structure in the form (adapted to the present problem)

$$
\begin{aligned}
& \overline{v^{\dagger}(x, y, z)}=\overline{v_{0}^{\dagger}(x, y)} \frac{\cosh \left(z / z_{0}\right)}{\cosh \left[(h-\delta) / z_{0}\right]}, \\
& z_{0}=f L / N,
\end{aligned}
$$

(an overbar denotes time averaging, and a "dagger" means that the velocity has been rotated into a coordinate system following the local isobaths) where $\delta$ is the bottom boundary layer thickness and $\overline{v_{0}^{\dagger}(x, y)}$ is the time-mean along-isobath velocity at the top of the bottom boundary layer, i.e., at $z=-(h-\delta)$. The form of $z_{o}$ arises from scaling the vorticity equation (either linear or Ertel, for example), and $L$ is a representative horizontal length scale. $\overline{v_{0}^{\dagger}(x, y)}$ will henceforth be called "the near-bottom mean velocity." Merryfield et al. (2001) suggest using a horizontal length scale $L^{N}$, which is related to the typical mean flow associated with topographic rectification in their unforced frictionless model (see their Eq. 21). The cosh form in (6a) is consistent with the application of a rigid lid boundary condition and with the mean flow being bottom-trapped.

For the present problem, (6) is used as a starting point for estimating the mean flow vertical structure. One issue arises immediately, however, and that is the choice of horizontal length scale for the present problem. Several alternatives for the scale were considered, including taking it to be a constant for all runs, taking it to be related to the bottom slope, e.g., $L^{S}=h /|\nabla h|$, or using a blend of the topographic cross-shelf scale and of the alongshore wavelength. For the set of numerical runs used here, no choice was better than that using the topographic wavelength:

$$
z_{0}=z_{T}=c f \lambda / N
$$

where $c$ is an empirical constant. The pairing (6a) and (7) is evaluated using the 24 numerical model runs that reached a reasonably steady mean flow. The fit was tested at more than $1.2 \times 10^{6}$ vertical points, spanning more than 9,000 vertical model kilometers. The result is that $c=0.19$, and the parameterization (6a) with (7) accounts for $63 \%$ of the energy in mean along-isobath flow, given the calculated near-bottom flow $\overline{v_{0}^{\dagger}(x, y)}$.

Given that this fit uses the actual model outputs for $\overline{v_{0}^{\dagger}(x, y)}$, it may seem strange that it is no better than it is. There are at least two reasons. One is that the maximum mean 
alongshore velocity does not always fall at the top of the bottom boundary layer, e.g., Figure 3b: at $x=45 \mathrm{~km}$, the mean velocity grows stronger with height for the first $100 \mathrm{~m}$ above the bottom boundary layer, and only then becomes weaker. This differs strongly relative to the monotonic cosh function of (6). However, given that the form (6a) works as well as it does implies that, in most cases, the maximum mean along-isobath flow does occur near the top of the bottom boundary layer. Second, (and consistent with the first point) the isotachs often tilt offshore (e.g., Fig. 3a), as mentioned previously. This tilt suggests that a parameterization based on scaled distance from the bottom (not necessarily vertically) might do better. An attempt was thus made to scale offshore distance as $x^{\prime}=x f / N$ (as suggested by vorticity conservation) and then seek a parameterization that depends on scaled distance $\sqrt{\Delta z^{2}+\Delta x^{\prime 2}}$ from the bottom. This approach's performance was indistinguishable from form (6a), and so the simpler parameterization is retained.

\section{b. Near-bottom velocity}

For the barotropic equivalent of the present problem (B10), the mean depth-averaged along-isobath flow is estimated by

$$
\overline{v^{\dagger}}=-\operatorname{sign}\left(f h_{\eta}\right) \min \left(\left|v^{T}\right|,\left|v^{\omega}\right|,\left|v^{M}\right|\right)
$$

where

$$
\begin{aligned}
v^{T} & =-a_{T} \operatorname{sign}(f) h_{\eta} S D\left(u_{\perp}\right) L^{T} / h, \\
v^{\omega} & =-a_{\omega} f h_{\eta} S D\left(u_{\perp}\right)^{2} /\left(h \omega^{2}\right), \\
v^{M} & =-a_{M} f h_{\eta} S D\left(u_{\perp}\right)^{2} /(r \omega),
\end{aligned}
$$

$h_{\eta}$ is the bottom slope normal to the isobaths, and $r$ is a linearized bottom resistance coefficient. B10 uses fitting of (8) to numerical model results to obtain $\left(a_{T}, a_{\omega}, a_{M}\right)=$ $(0.23,0.55,0.23)$. These velocities are estimated in a $(\xi, \eta)$ coordinate system defined so that $\xi$ is along-isobath, and $\eta$ is perpendicular to the isobath (positive toward deeper water). $S D\left(u_{\perp}\right)$ is the standard deviation of the cross-isobath depth-averaged velocity, and $L^{T}$ is a length scale for the topographic irregularities (taken to be the dominant wavelength $\lambda$ for periodic topography). The limit (8c) represents traditional tidal rectification (e.g., Huthnance, 1973), while the (8b) case arises when topographic along-isobath length scales are comparable to or shorter than along-isobath particle excursions. The third case (8d) occurs in the strong-stirring limit where the eddy transport is not associated with phase offsets due to friction, but rather with stirring by finite-amplitude motions. Altogether, this parameterization accounts for $60 \%$ of the mean along-isobath current energy over a suite of 39 varied barotropic numerical model runs. Applying this same parameterization to the present problem (which has quadratic bottom stress) suggests the replacement of $r$ by $c_{D} S D\left(u_{\perp}\right)$, an approximation that assumes that $u_{\perp}$ is representative of near bottom velocity. An application of (8) to the depth-averaged velocity from a suite of 24 repeatable baroclinic 
numerical model runs does not perform too well: only $49 \%$ of the mean along-isobath energy is accounted for, and the parameterized mean flow is overall somewhat (about 20\%) too weak.

It is not surprising, of course, that a parameterization from a barotropic model would not apply well to a case that includes density stratification: topography couples the baroclinic and barotropic modes strongly, so that results ought to be substantially changed. For example, the strongly bottom-trapped mean flow in the stratified case cannot be accounted for in a purely barotropic model. Nonetheless, the physical reasoning that led to (8) still seems viable, so this system is used as a starting point for parameterizing the mean along-isobath flow at the top of the bottom boundary layer.

Perhaps the most obvious problem with the system (8) is that it does not account for bottom trapping. Because bottom trapping is expected to occur over a vertical scale $h_{B}=$ $b f \lambda / N$ (where $b$ is an as-yet unknown empirical constant that need not equal $c$ ), bottomintensification effectively makes the water column shallower (i.e., only the lower part of the water column moves). Thus, (8) is modified by replacing the actual water depth $h$ by

$$
h^{*}=\min \left(h, h_{B}\right) .
$$

The motivation for this choice is that, in deep water, where the trapping scale is less than the water depth, the vertical trapping scale applies because the velocity is substantially weaker at the surface than the bottom. In effectively shallower water, where the natural vertical trapping scale exceeds the actual water depth, the actual depth is the more appropriate scale.

Thus the proposed scaling for the near-bottom mean along-isobath velocity $\overline{v_{0}^{\dagger}}$ is

$$
\overline{v_{0}^{\dagger}}=-\operatorname{sign}\left(f h_{\eta}\right) \min \left(\left|v_{0}^{T}\right|,\left|v_{0}^{\omega}\right|,\left|v_{0}^{M}\right|\right)
$$

where

$$
\begin{aligned}
v_{0}^{T} & =-a_{0 T} \operatorname{sign}(f) h_{\eta} S D\left(u_{0 \perp}\right) L^{T} / h^{*}, \\
v_{0}^{\omega} & =-a_{0 \omega} f h_{\eta} S D\left(u_{0 \perp}\right)^{2} /\left(h^{*} \omega^{2}\right), \\
v_{0}^{M} & =-a_{0 M} f h_{\eta} S D\left(u_{0 \perp}\right) / c_{D} \omega .
\end{aligned}
$$

$S D\left(u_{0 \perp}\right)$ is the standard deviation of the cross-isobath near-bottom velocity. This parameterization is tested (and the coefficients evaluated via least squares) using the 24 stratified numerical model runs that were geometrically simple, and had reached a sufficiently steady mean state. The result is that the values $b=0.10$ and $\left(a_{0 T}, a_{0 \omega}, a_{0 M}\right)=(0.25,4.3,0.13)$ allow (10) to account for $64 \%$ of the mean along-isobath near-bottom $\left(\overline{v_{0}^{\dagger}}\right)$ energy (Fig. 5). Of the three cases, (10b) applies at $47 \%$ of the points, (10c) at $15 \%$ of the points, and (10d) at $38 \%$ of the points. Results of the fit are the same when the one run with straight isobaths (670) is excluded from the fit. 


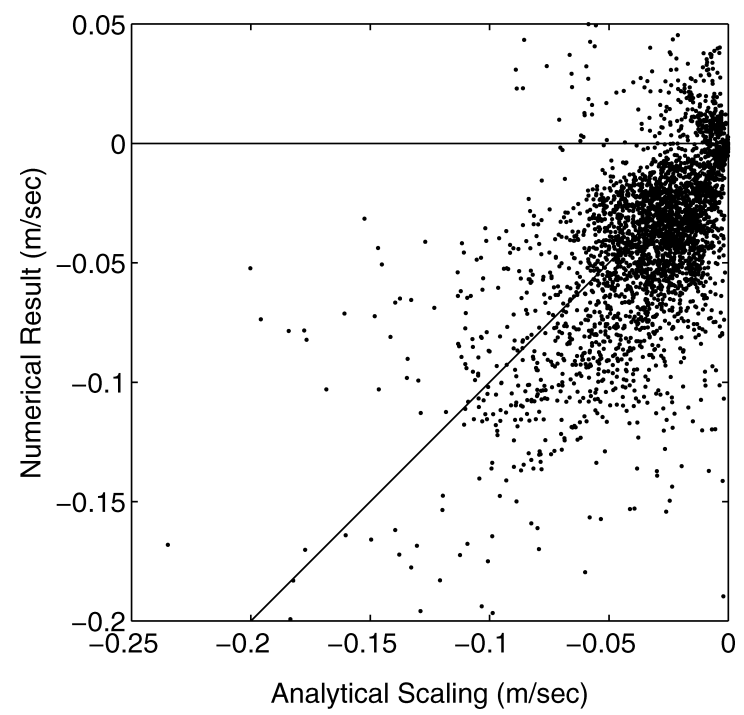

Figure 5. Scatter plot showing numerical model results for time-mean along-isobath velocity just above the bottom boundary layer (vertical axis), and scaling results (Eqs. 10) on the horizontal axis.

It should not be surprising that the fit coefficients $a_{0 T}, a_{0 \omega}$, and $a_{0 M}$ do not agree with those for the barotropic case. First, the baroclinic fit, through the contingent value of depth, takes into account the ocean's stratification, hence the tendency for mean currents to be bottomintensified. One implication of this is that the maximum mean along-isobath near-bottom currents in a stratified ocean might be expected to be stronger than depth-averaged currents in a barotropic case. Second, topographic rectification with stratification is inherently more complex. For example, topographic rectification associated with eddy momentum transports (Eq. 1b) is potentially supplemented by eddy heat transports (Eq. 1d) and skew fluxes (e.g., Loder and Horne, 2010) that can presumably affect the mean flow.

\section{Discussion}

The approximate time-mean along-isobath velocity just above the bottom boundary layer, $\overline{v_{0}^{\dagger}}$, is parameterized by expressions (9) and (10), and its overlying vertical structure is given by (6). The formulation builds on the barotropic results of B10, but the tendency for bottom trapping is now accounted for. This intensification of rectified mean momentum near the bottom, in many cases, leads to stronger near-bottom mean flows than would be expected from the barotropic case.

For mean along-isobath flow in a stratified ocean that has a sloping bottom, one might expect that down-slope density advection in the bottom boundary layer would eventually lead to buoyancy arrest, i.e., that the bottom velocity would be brought to rest geostrophically 
(e.g., MacCready and Rhines, 1991; Brink and Lentz, 2010a). Existing one-dimensional models of arrest (Brink and Lentz, 2010b) show that the presence of a superimposed fluctuating flow does not inhibit the tendency for lower-frequency (or steady) flow components to reach arrest. Yet, no examples of either steady or unsteady buoyancy arrest are found in any of the numerical model runs used for this study: the proportionality constant between interior flow and bottom stress remains finite and constant within a run. A closer look at conditions under the core of the upper slope jet for run 630 (Fig. 3a) shows that near-bottom vertical and cross-isobath "eddy" density transport counteracts the downslope advection associated with the mean flow. The net result is that the cross-isobath density gradient in the bottom boundary layer remains relatively constant over time, and no buoyancy arrest occurs.

The present model results are meant mainly to demonstrate how topographic rectification behaves in a stratified problem, and to establish which physical factors, such as time and length scales, are important. There is admittedly a difficulty in applying the present results because the real ocean does not usually have uniform stratification or well-defined sinusoidal forcings or topographies. Instead, there is always a continuum of scales present, so it is likely that measures such as correlation scales (e.g., B10) will be more relevant than wavelengths or periods. Further, in the real ocean, the forcing and ambient currents are expected to vary in space as well as time, and these forcing length scales also affect the rectified flow (B10 Appendix B). Thus, the present model results have to be taken as only crudely applicable to the real ocean.

Nonetheless it is intriguing to compare, however crudely, the present results to examples of observed oceanic flow in the downwave sense.

Fieberling Guyot: This steep-sided, flat-topped seamount lies in the North Pacific $\left(32.5^{\circ} \mathrm{N}\right)$ and was the site of intensive moored current measurements during 1990-1991 (e.g., Brink, 1995). The dominant current variability is the nearly resonant $K_{1}$ tide, and there is a localized mean along-isobath flow just above the rim of the guyot. Important parameter estimates are $L^{T}=30 \mathrm{~km}$ (the circumference of the flat summit), $N=3 \times 10^{-3} 1 / \mathrm{s}$, $\omega=7.3 \times 10^{-5} 1 / \mathrm{s}$ (the tidal frequency), $c_{D}=3 \times 10^{-3}$, and $S D\left(u_{0 \perp}\right)=0.01-0.03 \mathrm{~m} / \mathrm{s}$. Stronger tidal currents than this are found over the flat summit, and measurements at the summit depth over the flank may not be useful because they are from about $1000 \mathrm{~m}$ above the local bottom. The most remarkable parameter here is the slope of the seamount flanks, $h_{\eta}=0.36$. Brink (1995) provides some observational evidence for the mean currents being due to rectification, viz., the mean flow is correlated with the fortnightly tidal modulations. Finally, Brink (1995) detected a substantial radial tidal eddy heat transport consistent with the rectified flow structure. For the parameters above, the weakest estimated mean flow from Eqs. (10b-10d) is $v_{0}^{M}(0.04-0.2 \mathrm{~m} / \mathrm{s})$ and the lesser of the two depths (Eq. 9) is $h_{B}$ at $80 \mathrm{~m}$. The maximum observed mean along-isobath flow near the rim-flank junction is about $0.12 \mathrm{~m} / \mathrm{s}$, and it is distinctly bottom-trapped with a scale less than about $100 \mathrm{~m}$ (not inconsistent with $h_{B}$ ). In summary, the observed maximum mean along-isobath velocity of $0.12 \mathrm{~m} / \mathrm{s}$ is in range of present model estimates $\left(v_{0}^{M}=0.04-0.2 \mathrm{~m} / \mathrm{s}\right)$. 
Washington Continental Slope: One of the classic examples of a continental slope undercurrent is given by Hickey (1989) based on measurements offshore of the state of Washington. She finds a bottom-intensified poleward mean flow over the slope of $0.12-0.16 \mathrm{~m} / \mathrm{s}$ despite prevailing equatorward winds. Important parameters in this case include a bottom slope $h_{\eta}=0.06, S D\left(u_{0 \perp}\right)=0.024 \mathrm{~m} / \mathrm{s}$ (from Huyer et al., 1975), $N=3 \times 10^{-3} 1 / \mathrm{s}$ and $L^{T}=40 \mathrm{~km}$ from Hickey's Figure 2.13. As usual, the topographic scale $L^{T}$ is rather uncertain. Applying the present model to this case shows that the minimal mean velocity estimate is from Eq. $10 \mathrm{~b}$, i.e., $v_{0}^{T}=0.1 \mathrm{~m} / \mathrm{s}$, and that $h_{B}=130 \mathrm{~m}$ over the slope. It seems reasonable to conclude that the present model does not perform too badly ( 0.1 vs observed $0.12-0.16 \mathrm{~m} / \mathrm{s}$ ) here.

These comparisons with observations show that the present model (Eq. 10) estimates observed mean currents over topography to within the considerable uncertainty in the scaling fit itself (Fig. 5) and in the model parameters. This crude evaluation cannot be taken as a demonstration that the model works, but rather that it has potential. These comparisons highlight the need for developing a means to characterize the actual space and frequency scales that allow use of the model in a world that is not spatially or temporally monochromatic.

Acknowledgments. This work was sponsored by the Physical Oceanography Program at the National Science Foundation through grant OCE-0751731. Sam Wilson helped greatly with the bottom boundary layer elimination scheme. Comments from Steve Lentz are greatly appreciated.

My interest in this subject was first excited by working with Melvin Stern while I was a student in the Geophysical Fluid Dynamics program at Woods Hole in 1974.

\section{REFERENCES}

Brink, K. H. 1995. Tidal and lower frequency currents above Fieberling Guyot. J. Geophys. Res., 100, $10817-10832$.

2010. Topographic rectification in a forced, dissipative, barotropic ocean. J. Mar. Res., 68 , 337-368.

Brink, K. H. and S. J. Lentz. 2010a. Buoyancy arrest and bottom Ekman transport, Part I, Steady flow. J. Phys. Oceanogr., 40, 621-635.

2010b. Oscillating boundary layers over a sloping bottom. J. Phys. Oceanogr., 40, 636-655.

Chen, C. and R. C. Beardsley. 1995. A numerical study of stratified tidal rectification over finiteamplitude banks. Part I: Symmetric banks. J. Phys. Oceanogr., 25, 2090-2110.

Hickey, B. M. 1989. Patterns and processes of circulation over the Washington continental shelf and slope, in Coastal Oceanography of Washington and Oregon, M. R. Landry and B. M. Hickey, eds., Elsevier Science Publishers, Amsterdam, 41-115.

Holloway, G. 2008. Observing global ocean topostrophy. J. Geophys. Res., 113, C07054, doi:10.1029/2007JC004635.

Huthnance, J. M. 1973. Tidal current asymmetries over the Norfolk Sandbanks. Estuar. Coast. Mar. Sci., 1, 89-99.

Huthnance, J. M. 1978. On coastal trapped waves: analysis and numerical calculation by inverse iteration. J. Phys. Oceanogr., 8, 74-92.

Huyer, A., B. M. Hickey, J. D. Smith, R. L. Smith and R. D. Pillsbury. 1975. Alongshore coherence at low frequencies in currents observed over the continental shelf off Oregon and Washington. J. Geophys. Res., 80, 3495-3505. 
Loder, J. H. 1980. Topographic rectification of tidal currents on the sides of Georges Bank. J. Phys. Oceanogr., 10, 1399-1416.

Loder, J. H. and E. P. W. Horne. 2010. Skew eddy fluxes as signatures of non-linear tidal current interactions, with application to Georges Bank. Atmos.-Ocean, 29, 517-546.

Maas, L. R. M. and J. T. F. Zimmerman. 1989a. Tide-topography interactions in a stratified shelf sea I. Basic equations for quasi-nonlinear internal waves. Geophys. Astro. Fluid Dyn., 45, 1-35.

1989b. Tide-topography interactions in a stratified shelf sea II. Bottom trapped internal tides and baroclinic currents. Geophys. Astro. Fluid Dyn., 45, 37-69.

MacCready, P., and P. B. Rhines. 1991. Buoyant inhibition of Ekman transport on a slope and its effect on stratified spin-up. J. Fluid Mech., 223, 631-661.

Merryfield, W. J. 1998. Effects of stratification on quasi-geostrophic inviscid equilibria. J. Fluid Mech., 354, 345-356.

Merryfield, W. J., P. F. Cummins and G. Holloway. 2001. Equilibrium statistical mechanics of barotropic flow over finite topography. J. Phys. Oceanogr., 31, 1880-1890.

Samelson, R. M. and J. S. Allen. 1987. Quasi-geostrophic topographically generated mean flow over the continental margin. J. Phys. Oceanogr., 17, 2043-2094.

She, J. and J. M. Klinck. 2000. Flow near canyons driven by constant winds. J. Geophys. Res., 105, 28,671-28,694.

Shchepetkin, A. F. and J. C. McWilliams. 2005. The regional oceanic modeling system (ROMS): A split-explicit, free-surface, topography-following-coordinate oceanic model. Ocean Model., 9 , 347-404.

Stern, M. E. and C. Y. Shen. 1976. Displacement and rectification of planetary fluids. Geophys. Fluid Dyn., 7, 81-118.

Visser, A.W. 1994. On tidal rectification, friction and geostrophic degeneracy. J. Phys. Oceanogr., 24, 2196-2200.

Wijesekera, H. W., J. S. Allen and P. Newberger. 2003. A modeling study of turbulent mixing over the continental shelf: Comparison of turbulent closure schemes. J. Geophys. Res., 108 (C3), 3103, doi:10.1029/2001JC001234.

Wright, D. G. and J.W. Loder. 1988. On the influences of nonlinear bottom friction on the topographic rectification of tidal currents. Geophys. Astro. Fluid Dyn., 42, 227-245.

Received: 3 March, 2011; revised: 26 July, 2011. 